\title{
Visibility Transition Planning for Dynamic Camera Control
}

\author{
Thomas Oskam ${ }^{1}$, Robert W. Sumner ${ }^{2}$, Nils Thuerey ${ }^{1}$, and Markus Gross ${ }^{1,2}$ \\ 1 ETH Zurich, Switzerland \\ 2 Disney Research Zurich, Switzerland
}

\begin{abstract}
We present a real-time camera control system that uses a global planning algorithm to compute large, occlusion free camera paths through complex environments. The algorithm incorporates the visibility of a focus point into the search strategy, so that a path is chosen along which the focus target will be in view. The efficiency of our algorithm comes from a visibility-aware roadmap data structure that permits the precomputation of a coarse representation of all collision-free paths through an environment, together with an estimate of the pair-wise visibility between all portions of the scene. Our runtime system executes a path planning algorithm using the precomputed roadmap values to find a coarse path, and then refines the path using a sequence of occlusion maps computed on-the-fly. An iterative smoothing algorithm, together with a physically-based camera model, ensures that the path followed by the camera is smooth in both space and time. Our global planning strategy on the visibility-aware roadmap enables large-scale camera transitions as well as a local third-person camera module that follows a player and avoids obstructed viewpoints. The data structure itself adapts at runtime to dynamic occluders that move in an environment. We demonstrate these capabilities in several realistic game environments.
\end{abstract}

